\title{
CAUCHY-RIEMANN VECTOR FIELDS
}

\author{
W. H. GREUB and J. R. VANSTONE
}

Introduction. It is the purpose of this paper to introduce the notions of (i) a Cauchy-Riemann vector field (CR-field) on a 2-dimensional Riemannian manifold, (ii) the index of a line field via mapping degree (cf. the definition in H. Poincaré [4], Ch. XIII) and to use these to obtain a modernized proof of a classical result of $H$. Hopf [3].

In $\S 1$ we discuss the general properties of CR-fields. In particular, it is shown that a nonzero $\mathrm{CR}$-field has only isolated zeros.

One of the main results of $\S 2$ states that if $Z$ is a CR-field with an isolated singularity at a point $a$ and $\lim _{x \rightarrow a}|Z(x)|=\infty$, then the index of $Z$ at $a$ is negative.

The Gauss-Bonnet theorem for a line field on a compact oriented Riemannian 2-manifold is established in $\S 3$.

Finally, in $\S 4$ we apply the above notions and results to give a simple proof of a theorem of H. Hopf on immersions of 2 -spheres in $\boldsymbol{R}^{\mathbf{3}}$ with constant mean curvature.

\section{Cauchy-Riemann vector fields}

1.1. Cauchy-Riemann vector fields. Let $M$ be a smooth orientable 2-manifold.

We shall denote the ring of smooth functions on $M$ by $\subseteq(M)$ and the $\mathfrak{S}(M)$-module of vector fields on $M$ by $\mathfrak{X}(M)$ (cf. [2] for details).

Recall that an almost complex structure on $M$ is a tensor field $J$ of type $(1,1)$ such that $J^{2}=-I$. In particular, a Riemannian metric $g$ on an oriented 2-manifold determines an almost complex structure given by

$$
g(J X, Y)=\Delta_{M}(X, Y), \quad X, Y \in \mathfrak{X}(M),
$$

where $\Delta_{M}$ denotes the normed 2 -form on $M$ which represents the orientation. 
A vector field $Z$ on $M$ will be called a Cauchy-Riemann field (CRfield) if, for every $X \in \mathfrak{X}(M)$,

$$
J([Z, X])=[Z, J X] .
$$

Example. Let $M=C$ (the complex plane) and consider a vector field

$$
Z=u e_{1}+v e_{2}, \quad u, v \in \mathbb{S}(C),
$$

where $e_{1}, e_{2}$ is a positive orthonormal basis of $C$. Then it is easily checked that $Z$ is a CR-field if and only if $u$ and $v$ satisfy the CauchyRiemann conditions

$$
e_{1}(u)=e_{2}(v), \quad e_{1}(v)=-e_{2}(u) .
$$

$\mathrm{L}$ e $\mathrm{m} \mathrm{m}$ a I. A vector field $Z$ on $M$ is a CR-field if and only if

$$
[Z, J Z]=0 .
$$

Proof. It is obvious that (1.2) follows from (1.1). Conversely, assume that (1.2) holds. Fix a point $a \in M$. We may assume that $a \in \operatorname{carr} Z$. Now we distinguish two cases:

Case I. $Z(a) \neq 0$. Then $Z(x) \neq 0$ in some neighbourhood $U$ of $a$ and so the vector fields $Z$ and $J Z$ determine an orthogonal 2 -frame at every point $x \in U$. Thus, if $X \in \mathfrak{X}(M)$, we have in $U$

$$
X=\alpha Z+\beta J Z, \quad J X=-\beta Z+\alpha J Z, \quad \alpha, \beta \in \subseteq(U),
$$

and so (1.2) implies that

$$
[Z, X]=[Z, \alpha Z]+[Z, \beta J Z]=Z(\alpha) \cdot Z+Z(\beta) \cdot J Z,
$$

whence

$$
J([Z, X])=Z(\alpha) \cdot J Z-Z(\beta) \cdot Z=[Z, J X], \quad \text { in } U .
$$

In particular,

$$
J[Z, X](a)=[Z, J X](a) .
$$

Case II. $Z(a)=0$. Then (1.1) follows from Case I via a continuity argument since $a \in \operatorname{carr} Z$.

Coroll a ry. If $Z$ is a CR-field, then so is $J Z$.

From now on we will assume that the almost complex structure on $M$ is induced by a Riemannian metric $g$. Then we have $\nabla_{X} J=0$, where $\nabla_{X}$ denotes covariant differentiation in the direction of the vector field $X$ with respect to the corresponding Levi-Civita connection. Note that if $g$ is replaced by $\lambda \cdot g$ ( $\lambda$ a positive function on $M$ ), then $J$ is not changed and hence a CR-field in the $g$-metric is also a CR-field in the $\lambda \cdot g$-metric. 
$\mathrm{L}$ e m m a I I. With the above notation, a vector field $Z$ is a $C R$-field if and only if

$$
\nabla_{J X} Z=J \nabla_{X} Z, \quad X \in \mathfrak{X}(M) .
$$

In particular, a parallel vector field is a $C R$-field.

Proof. Since $\nabla$ is torsion free we have for any two vector fields $X$ and $Z$

$$
\nabla_{Z} X-\nabla_{X} Z=[Z, X]
$$

This relation yields

$$
\nabla_{Z} J X-J \nabla_{X} Z=J[Z, X]
$$

and

$$
\nabla_{Z} J X-\nabla_{J X} Z=[Z, J X]
$$

It follows that

$$
\nabla_{J X} Z-J \nabla_{X} Z=J[Z, X]-[Z, J X] .
$$

Thus $Z$ is a CR-field if and only if (1.3) holds.

1.2. The 1 -forms $\Phi_{Z}$ and $\Psi_{Z}$. Let $Z$ be a vector field on $M$ without zeros. Then we can write

$$
\nabla_{X} Z=\Phi_{Z}(X) \cdot Z+\Psi_{Z}(X) \cdot J Z, \quad X \in \mathfrak{X}(M),
$$

where $\Phi_{Z}$ and $\Psi_{Z}$ are 1-forms on $M$. They are given explicitly by

$$
\Phi_{Z}(X)=X(\ln |Z|)
$$

and

$$
\Psi_{Z}(X)=\frac{1}{|Z|^{2}} \Delta_{M}\left(Z, \nabla_{X} Z\right)
$$

Proposition I. The exterior derivatives of $\Phi_{Z}$ and $\Psi_{Z}$ are given by

$$
\delta \Phi_{Z}=0
$$

and

$$
\delta \Psi_{Z}=-K \Delta_{M},
$$

where $K$ denotes the Gaussian curvature of $M$.

Proof. Let $R$ denote the curvature operator of $M$ on $\mathfrak{X}(M)$ :

$$
R(X, Y)=\nabla_{X} \nabla_{Y}-\nabla_{Y} \nabla_{X}-\nabla_{[X, Y]}, \quad X, Y \in \mathfrak{X}(M),
$$

(cf. [2], p. 321). Then (1.4) yields after a short calculation,

$$
R(X, Y) Z=\delta \Phi_{Z}(X, Y) \cdot Z+\delta \Psi_{Z}(X, Y) \cdot J Z .
$$


On the other hand, the Gaussian curvature is determined by the equation

$$
R(X, Y) Z=-K \Delta_{M}(X, Y) \cdot J Z .
$$

Now the proposition follows from (1.9) and (1.10).

Proposition II. A vector field $Z$ without zeros is a CR-field if and only if the 1-forms $\Phi_{Z}$ and $\Psi_{Z}$ satisfy

$$
* \Phi_{Z}=\Psi_{Z}
$$

where * denotes the Hodge star operator (cf. [5], p. 121).

Proof. First recall that, for any 1-form $\Phi$ on a Riemannian 2-manifold, $* \Phi(X)=-\Phi(J X), \quad X \in \mathfrak{X}(M)$. Now let $Z \in \mathfrak{X}(M)$. Then (1.4) yields

$$
\nabla_{J X} Z-J \nabla_{X} Z=\left[\Phi_{Z}(J X)+\Psi_{Z}(X)\right] Z+\left[\Psi_{Z}(J X)-\Phi_{Z}(X)\right] J Z \text {. }
$$

Thus $Z$ is a CR-field if and only if $\Psi_{Z}(X)=-\Phi_{Z}(J X)=* \Phi_{Z}(X)$; i.e., $\Psi_{Z}=* \Phi_{Z}$.

Corollary I. Let $Z$ be a CR-field without zeros. Then

$$
\Delta \ln |Z|=K \text {. }
$$

Proof. In fact, by definition of the (Hodge) Laplacian (cf. [5], p. 125)

$$
\Delta \ln |Z|=-* \delta * \delta \ln |Z|=-* \delta * \Phi_{Z} .
$$

If $Z$ is a CR-field, Propositions I and II yield

$$
\Delta \ln |Z|=-* \delta \Psi_{Z}=K * \Delta_{M}=K .
$$

Corollary II. If $Z$ is a CR-field without zeros, then

$$
\Delta|Z|^{p}=p K|Z|^{p}-p^{2}|Z|^{p-2}|\delta| Z||^{2}, \quad p=1,2, \ldots .
$$

Proof. Apply the formula $e^{-f} \Delta\left(e^{f}\right)=\Delta f-|\delta f|^{2}$, when $f=p \ln |Z|$. Cor o Il a r y I I I. A CR-field, $Z$, with constant length is parallel.

Proof. We may assume that the constant length is positive. Then, by (1.5), $\Phi_{Z}=0$. Thus Proposition II implies that $\Psi_{Z}=0$, whence $\nabla_{X} Z=0, \quad X \in \mathfrak{X}(M)$.

1.3. Existence of CR-vector fields. In this section we shall prove the local existence of non-trivial CR-fields.

Proposition I I L. Let $a \in M$ and let $h \in T_{a}(M)$ be a nonzero tangent vector. Then there is a CR-field $Z$ in some neighbourhood $U$ of a such that $Z(a)=h$.

Proof. Assume first that the Gaussian curvature $K$ vanishes in a neighbourhood $V$ of $a$. Choose a simply connected open subset $U \subset V$ containing $a$. Then there is precisely one parallel vector field $Z$ in $U$ such that $Z(a)=h$. By Lemma II, $Z$ is a CR-field in $U$. 
In the general case introduce a new Riemannian metric $\tilde{g}$ on $M$ by setting

$$
\tilde{g}=e^{2 \lambda} g, \quad \lambda \in \subseteq(M) .
$$

The corresponding Gaussian curvature $\tilde{K}$ is given by

$$
e^{2 \lambda} \tilde{K}=K+\Delta \lambda
$$

where $\Delta$ denotes the Laplacian with respect to the metric $g$. Choose for $\lambda$ a local solution of the elliptic differential equation

$$
\Delta \lambda=-K
$$

(cf. [5], p. 151). Then $\tilde{K}=0$ and so there exists a local CR-field $Z$ in the $\tilde{g}$-metric such that $Z(a)=h$. But this is also a CR-field in the $g$-metric. (Cf. the remark above Lemma II.)

1.4. Cauchy-Riemann frames. Let $e_{1} \neq 0$ be a CR-field in a simply connected neighbourhood $U$ of a point $a \in M$ and set $e_{2}=J e_{1}$. Then $e_{2}$ is again a $\mathrm{CR}$-field (cf. corollary to Lemma I). Moreover, we have the relations

$$
\left|e_{1}\right|=\left|e_{2}\right|, \quad g\left(e_{1}, e_{2}\right)=0 \text { and }\left[e_{1}, e_{2}\right]=0 .
$$

Thus $e_{1}, e_{2}$ is an orthogonal frame field in $U$. It will be called a CauchyRiemann frame (CR-frame).

Now consider the dual frame $e^{* 1}, e^{* 2}$. Then

$$
\delta e^{* 1}=0 \quad \text { and } \quad \delta e^{* 2}=0 .
$$

Thus $e^{* 1}$ and $e^{* 2}$ are gradient fields,

$$
e^{* i}=\delta x^{i}, \quad x^{i} \in \mathfrak{S}(U), \quad i=1,2 .
$$

Since the covectors $e^{* 1}(x)$ and $e^{* 2}(x)$ are linearly independent, it follows that the functions $x^{1}, x^{2}$ are local coordinates in a neighbourhood $V \subset U$ of $a$. In this local coordinate system the metric tensor satisfies $g_{11}=g_{22}$, $g_{12}=0$ and so $\left(x^{1}, x^{2}\right)$ is a system of isothermal parameters.

Now choose a covering $M=\mathrm{U}_{\alpha} V_{\alpha}$ by such open sets and introduce, in each $V_{\alpha}$, isothermal parameters. Then it is easy to check that corresponding indentification maps are conformal and so $M$ becomes a 1-dimensional complex analytic manifold.

Finally, let $e_{1}, e_{\mathbf{2}}$ be a CR-frame in $U$ and let

$$
Z=u e_{1}+v e_{2}
$$

be a vector field. Then it is easy to check that $Z$ is a CR-field if and only if the functions $u$ and $v$ satisfy 


$$
e_{1}(u)=e_{2}(v), \quad e_{1}(v)=-e_{2}(u) .
$$

Thus if $Z$ is a CR-field, then

$$
f=u+i v
$$

is a complex analytic function in $U$.

In particular, a nonzero CR-field has only isolated zeros.

\section{The index of a line field at an isolated singularity}

2.1. The index of a vector field. Let $X$ be a vector field on an oriented 2-manifold $M$ with an isolated singularity at a point $a$. Recall that the index of $X$ at $a$ is defined as follows: Choose a local trivialization of the tangent bundle, $T_{U} \stackrel{\cong}{\rightarrow} U \times \mathbb{R}^{2}$. Then $X$ determines a map $X_{U}: \dot{U}=U-\{a\} \rightarrow \dot{R}^{2}\left(=\mathbb{R}^{2}-\{0\}\right) ;$ the index of $X$ at $a$ is the local degree of $X_{U}$ at $a$,

$$
j_{a}(X)=\operatorname{deg}_{a} X_{U} .
$$

It is well known that the index can be expressed in terms of a line integral. Regard $R^{2}$ as the complex plane $C$ and let $\Omega$ denote the 1 form in $\dot{C}=C-\{0\}$ given by

$$
\Omega(z ; h)=\frac{1}{|z|^{2}} \Delta(z, h)=\frac{1}{|z|^{2}} \operatorname{Im}(\bar{z} h), \quad z \in \dot{C}, h \in C,
$$

where $\Delta$ is the normed determinant function. Then

$$
j_{a}(X)=\frac{1}{2 \pi} \int_{S_{a}} X_{U}^{*} \Omega,
$$

where $S_{a}$ is a positively oriented 1-sphere in $U$ around $a$.

If $e_{1}, e_{2}$ is a positively oriented 2 -frame in $U$, then the corresponding trivialization of $T_{U}$ is given by $X(x) \mapsto\left(x,\left[e^{* 1}(X)+i e^{* 2}(X)\right](x)\right)$, $x \in U$, where $e^{* 1}, e^{* 2}$ is the dual 2-frame. In particular, if $X=$ $u e_{1}+v e_{2}$, then $X_{U}=u+i v$.

Now formula (2.2) reads

$$
j_{a}(X)=\frac{1}{2 \pi} \int_{S_{a}} \frac{u \delta v-v \delta u}{u^{2}+v^{2}} .
$$

Proposition I. Let $Z$ be a CR-field with an isolated singularity at $a$. Then 


$$
j_{a}(Z)\left\{\begin{array}{rrr}
>0, & \text { if } \quad \lim _{x \rightarrow a}|Z|=0 ; \\
=0, & \text { if } & 0<\lim _{x \rightarrow a}|Z|<\infty ; \\
<0, & \text { if } & \lim _{x \rightarrow a}|Z|=\infty .
\end{array}\right.
$$

Proof. Choose a CR-frame $e_{1}, e_{2}$ in a neighbourhood $U$ of $a$ and write

$$
Z=u e_{1}+v e_{2}
$$

Then

$$
f=u+i v
$$

is a complex analytic function in $U$ (cf. section 1.4) and so we have, in view of $(2.3)$,

$$
j_{a}(Z)=\frac{1}{2 \pi i} \int_{S_{a}} \frac{f^{\prime}(z)}{f(z)} d z .
$$

Now the proposition follows from a standard theorem on complex analytic functions.

2.2. Line fields. Let $M$ be a smooth oriented 2-manifold with tangent bundle $\tau_{M}=\left(T_{M}, p, M, R^{2}\right)$ and consider the corresponding projective bundle $\pi_{M}=\left(P_{M}, q, M, R P^{1}\right)$ whose fibre at $x$ consists of the 1-dimensional subspaces of $T_{x}(M)$. A line field on $M$ is a smooth cross-section in $\pi_{M}$.

Suppose now that $\sigma$ is a line field on $M$ with an isolated singularity at a point $a$. To define the index of $\sigma$ at $a$, choose a local trivialization $P_{U} \stackrel{\cong}{\rightarrow} U \times \boldsymbol{R} P^{1}$ of $\pi_{M}$ and consider the map $\sigma_{U}: \dot{U} \rightarrow \boldsymbol{R} P^{1}$ determined by $\sigma$. The index of $\sigma$ at $a$ is defined as the mapping degree

$$
j_{a}(\sigma)=\operatorname{deg}_{a} \sigma_{U} .
$$

In particular, if the line field $\sigma$ is induced from a unit vector field $X$ with an isolated singularity at $a$, then $\sigma_{U}=\varrho \circ X_{U}$, where $\varrho: S^{1} \rightarrow R P^{1}$ is the double covering. Thus,

$$
j_{a}(\sigma)=2 j_{a}(X) .
$$

Let $\varphi: M \rightarrow N$ be a smooth map between 2 -manifolds such that the induced map $(d \varphi)_{x}$ is a linear isomorphism for each $x \in M$. Then $\varphi$ induces a bundle map $\tilde{\varphi}: P_{M} \rightarrow P_{N}$ which restricts to diffeomorphisms on the fibres. Thus every line field $\tau$ on $N$ determines a line field $\sigma$ on $M$ given by

$$
\sigma(x)=\tilde{\varphi}(x)^{-1} \tau(\varphi(x)), \quad x \in M .
$$


Moreover, if $\tau$ has an isolated singularity at $b=\varphi(a)$, then $\sigma$ has an isolated singularity at $a$ and

$$
j_{a}(\sigma)=\operatorname{deg}_{a} \varphi \cdot j_{b}(\tau),
$$

as follows from standard properties of the mapping degree.

As in the case of vector fields there is an integral formula for $j_{a}(\sigma)$. In fact, consider the double covering $\varrho: S^{1} \rightarrow R P^{1}$ and let $\Omega_{R P^{1}}$ be the unique 1 -form on $R P^{1}$ satisfying

$$
\varrho^{*} \Omega_{R P^{1}}=\Omega \text {. }
$$

Since $\varrho$ has degree 2 , it follows that

Thus

$$
\int_{R P^{1}} \Omega_{R P^{1}}=\pi
$$

$$
\operatorname{deg}_{a} \sigma_{U}=\frac{1}{\pi} \int_{S_{a}} \sigma_{U}^{*} \Omega_{R P^{1}}
$$

and we obtain the formula

$$
j_{a}(\sigma)=\frac{1}{\pi} \int_{S_{a}} \sigma_{U}^{*} \Omega_{R P^{1}}
$$

2.3. Proposition I I. Let $\varphi$ be a smooth map from a connected 2-manifold $M$ to $S^{1}$. Assume that the induced map in homology takes all elements of $H_{1}(M ; \boldsymbol{Z})$ into even multiples of the generator of $H_{1}\left(S^{1} ; \boldsymbol{Z}\right)$. Then there is a smooth map $\psi: M \rightarrow S^{1}$ such that

$$
\varphi(x)=\psi(x)^{2}, \quad x \in M .
$$

Proof. Choose a base point $x_{0}$ on $M$. Without loss of generality we may assume that $\varphi\left(x_{0}\right)=1$. Let $\Omega$ be the 1-form on $S^{1}$ given by (2.1). The 1-form $\Omega$ determines a 1 -form $\Omega_{\varphi}=\varphi^{*} \Omega$ on $M$. If $\alpha$ is a loop on $M$ we have, in view of the hypothesis,

$$
\int_{\alpha} \Omega_{\varphi}=\int_{\alpha} \varphi^{*} \Omega=\int_{\varphi(\alpha)} \Omega=2 k \int_{S^{1}} \Omega=4 k \pi, \quad k \in Z .
$$

Thus a smooth map $\psi: M \rightarrow S^{1}$ is well defined by

$$
\psi(x)=\exp \left(\frac{i}{2} \int_{x_{0}}^{x} \Omega_{\varphi}\right), \quad x \in M .
$$


To show that $\psi(x)^{2}=\varphi(x)$, consider the map $\chi: M \rightarrow S^{1}$ given by

$$
\chi(x)=\psi(x)^{2}=\exp \left(i \int_{x_{0}}^{x} \Omega_{\varphi}\right) .
$$

It satisfies

$$
(d \chi)_{x} h=\Omega_{\varphi}(x ; h) i \chi(x), \quad x \in M, h \in T_{x}(M) .
$$

On the other hand, the relation $(\varphi(x), \varphi(x))=1, x \in M$, implies that

$$
(d \varphi)_{x} h=\Omega\left(\varphi(x) ;(d \varphi)_{x} h\right) i \varphi(x)=\Omega_{\varphi}(x ; h) i \varphi(x) .
$$

Equations (2.6) and (2.7) show that

$$
\chi=\lambda \cdot \varphi, \quad \lambda \in C .
$$

Since $\chi\left(x_{0}\right)=\varphi\left(x_{0}\right)=1$, it follows that $\chi=\varphi$.

Corollary. Let $\varphi: M \rightarrow R P^{1} \quad$ ( $M$ connected) be a smooth map and assume that the induced map in homology takes all the elements of $H_{1}(M: Z)$ into even multiples of the generator of $H_{1}\left(\boldsymbol{R} P^{1} ; Z\right)$. Then $\varphi$ lifts to a smooth map $\tilde{\varphi}: M \rightarrow S^{1}$.

Proof. Consider the diffeomorphism $\alpha: S^{1} \stackrel{R}{\cong} P^{1}$ which is determined by the commutative diagram

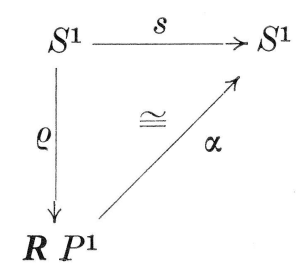

where $s(z)=z^{2}, z \in S^{1}$. Then the composite map

$$
M \stackrel{\varphi}{\longrightarrow} R P^{1} \stackrel{\alpha}{\longrightarrow} S^{1}
$$

satisfies the hypothesis of Proposition II. Thus there is a smooth map $\tilde{\varphi}: M \rightarrow S^{1}$ such that

$$
\alpha \circ \varphi=s \circ \tilde{\varphi}=\alpha \circ \varrho \circ \tilde{\varphi} .
$$

It follows that

$$
\varphi=\varrho \circ \tilde{\varphi} .
$$

Proposition III. Let $\sigma$ be a line field in a neighbourhood $U$ of $a$ with an isolated singularity at $a$. Then $\sigma$ lifts to a vector field if and only if $j_{a}(\sigma)$ is even. 
Proof. If $\sigma$ lifts to a vector field, formula (2.4) shows that $j_{a}(\sigma)$ is even. Conversely, if $j_{a}(\sigma)$ is even, choose a trivialization of $\tau_{U}$ and apply the corollary of Proposition II to $\sigma_{U}$.

\section{The Gauss-Bonnet theorem for line fields}

3.1. Line fields on Riemannian manifolds. In section 3.3 it will be shown that the index sum of a line field $\sigma$ with finitely many singularities on a compact oriented Riemannian 2-manifold $M$ is given by

$$
j_{\sigma}=\frac{1}{\pi} \int_{M} K \Delta_{M},
$$

where $K$ denotes the Gaussian curvature of $M$. The proof is essentially based on a 1-form $\Psi_{\sigma}$ associated with $\sigma$.

Consider the circle bundle $\left(S_{M}, r, M, S^{1}\right)$ associated with $\tau_{M}$ via the metric and observe that $S_{M}$ is a double covering manifold of $P_{M}$. Choose an open covering $M=\mathrm{U}_{\alpha} U_{\alpha}$ such that the covering projection $\varrho: S_{M} \rightarrow P_{M}$ admits a cross-section over each $U_{\alpha}$. Then there are precisely two unit vector fields $X_{\alpha}$ and $-X_{\alpha}$ in $U_{\alpha}$ such that

Now set

$$
\varrho^{\circ} X_{\alpha}=\sigma \text {. }
$$

$$
\Psi_{\alpha}(x ; h)=\Delta_{M}\left(x ; X_{\alpha}(x), \nabla X_{\alpha}(x ; h)\right), \quad x \in U_{\alpha}, h \in T_{x}\left(U_{\alpha}\right) .
$$

Since $\Psi_{\alpha}$ is not changed if $X_{\alpha}$ is replaced by $-X_{\alpha}$, it is a well-defined 1-form in $U_{\alpha}$. Moreover, if $U_{\alpha} \cap U_{\beta} \neq \varnothing$, we have

$$
\Psi_{\alpha}=\Psi_{\beta} \quad \text { in } U_{\alpha} \cap U_{\beta}
$$

and so the local 1-forms $\Psi_{\alpha}$ determine a 1-form $\Psi_{\sigma}$ on $M$.

Proposition I. The 1-form $\Psi_{\sigma}$ has the following properties:

(i) $\delta \Psi_{\sigma}=-K \Delta_{M}$;

(ii) if $\sigma$ has an isolated singularity at $a$ and if the metric is flat in a neighbourhood of $a$, then

$$
j_{a}(\sigma)=\frac{1}{\pi} \int_{S^{1}} \Psi_{\sigma} .
$$

Proof. (i) follows directly from the definition of $\Psi_{\sigma}$ and formula (1.8).

To establish (ii) observe that, since the metric is flat near $a$, we can choose a trivialization $T_{U} \cong U \times \boldsymbol{R}^{2}$ which induces isometries between the tangent spaces. It is easily checked that for such a trivialization 


$$
\sigma_{U}^{*} \Omega_{R P^{1}}=\Psi_{\sigma}
$$

and so (ii) follows from formula (2.5).

3.2. The Gauss-Bonnet theorem. Suppose now that $M$ is oriented and compact. Let $\sigma$ be a line field with finitely many singularities and denote the index sum of $\sigma$ by $j_{\sigma}$.

$\mathrm{T} \mathrm{h}$ e o $\mathrm{r}$ e $\mathrm{m}$. With the notation and hypotheses above,

$$
j_{\sigma}=\frac{1}{\pi} \int_{M} K \Delta_{M} .
$$

Thus $j_{\sigma}=2 \chi(M)$, where $\chi(M)$ denotes the Euler characteristic of $M$.

For the proof we establish first

$\mathrm{L}$ e m m a I. Let $g_{1}$ and $g_{2}$ be Riemannian metrics on an oriented 2-manifold which agree in a neighbourhood $U$ of a point $a$. Denote the corresponding normed 2-forms and Gaussian curvatures by $\Delta_{i}$ and $K_{i}$ ( $i=$ 1, 2 ). Then the 2-form

$$
K_{1} \Delta_{1}-K_{2} \Delta_{2}
$$

is exact on $M$.

Proof. Denote the Levi-Civita connections corresponding to $g_{i}$ by $\nabla_{i}(i=1,2)$. Choose a vector field, $X$, with a single singularity at $a$ and consider the 1 -forms

$$
\Psi_{i}(x ; h)=\frac{\Delta_{i}\left(x ; X(x), \nabla_{i} X(x ; h)\right)}{g_{i}(x ; X(x), X(x))}, \quad x \in M-\{a\}, i=1,2 .
$$

Then by (1.8)

$$
\delta \Psi_{i}=-K_{i} \Delta_{i}, \quad i=1,2,
$$

whence

$$
\delta\left(\Psi_{2}-\Psi_{1}\right)=K_{1} \Delta_{1}-K_{2} \Delta_{2} .
$$

But in $U$ we have $g_{1}=g_{2}$ and thus (3.1) holds on the whole manifold $M$.

3.3. Proof of the theorem. We first reduce to the case that $g$ is flat in a neighbourhood of the singularities $a_{i}(i=1, \ldots, r)$. Choose neighbourhoods $U_{i}$ of $a_{i}$ such that $U_{i} \cap U_{j}=\varnothing$ for $i \neq j$ and let $V_{i} \subset U_{i}$ be open subsets diffeomorphic to the unit disk such that $\bar{V}_{i} \subset U_{i}$. Set

Then

$$
U=M-\bigcup_{i=1}^{r} \bar{V}_{i}
$$

$$
M=U_{1} \cup \cdots \cup U, \cup U
$$


is an open covering of $M$. Choose a partition of unity (cf. [1], p. 32) $f_{1}, \ldots, f_{r}, f$ subordinate to this covering. Then

$$
\tilde{g}=f \cdot g+\sum_{i=1}^{n} f_{i} \cdot g_{i}
$$

is again a Riemannian metric where $g_{i}$ is a flat metric in $U_{i}$. Since $\tilde{g}=g_{i}$ in $V_{i}$, it follows that $\tilde{g}$ is flat in $V_{i}$. On the other hand, $\tilde{g}=g$ in $M-U_{i=1}^{r} \bar{U}_{i}$. Thus, by Lemma I,

$$
\int_{M} K \Delta=\int_{M} \tilde{K} \tilde{\Delta}
$$

Hence we may assume that $g$ is flat in $V_{i}(i=1, \ldots, r)$.

Now consider the 1 -form $\Psi_{\sigma}$ (cf. section 3.1) in the complement of the singularities $\left\{a_{1}, \ldots, a_{r}\right\}$. Set $V=\mathrm{U}_{i=1}^{r} V_{i}$. Then Stokes' theorem yields, in view of Proposition I, (i)

$$
\int_{M-V} K \Delta_{M}=\sum_{i=1}^{r} \int_{S_{a_{i}}} \Psi_{\sigma}, \quad S_{a_{i}}=\partial V_{i}, i=1, \ldots, r .
$$

On the other hand, by part (ii) of that proposition,

$$
\int_{S_{a_{i}}} \Psi_{\sigma}=\pi j_{a_{i}}(\sigma), \quad i=1, \ldots, r .
$$

Finally, since the metric is flat in $V$,

$$
\int_{V} K \Delta_{M}=0
$$

These equations yield

$$
\int_{M} K \Delta_{M}=\pi \sum_{i=1}^{r} j_{a_{i}}(\sigma)=\pi j_{\sigma} .
$$

\section{Immersions into $R^{3}$ with constant mean curvature}

4.1. Let $M$ be an oriented Riemannian 2-manifold and let $\varphi: M \rightarrow R^{3}$ be an isometric immersion. Let $F_{x}$ denote the oriented plane in $\boldsymbol{R}^{\mathbf{3}}$ given by

$$
F_{x}=(d \varphi)_{x} T_{x}(M), \quad x \in M .
$$

Then there is a unique unit vector $n(x) \in R^{3}$ orthogonal to $F_{x}$ such that the oriented plane $F_{x}$ together with $n(x)$ induces the given orientation of 
$\boldsymbol{R}^{\mathbf{3}}$. The correspondence $x \mapsto n(x)$ determines a smooth map $n: M \rightarrow \boldsymbol{R}^{3}$ called the normal field of the immersion $\varphi$.

Recall that the second fundamental form for $\varphi$ is the symmetric tensor field of degree two on $M$ given by

$$
\Lambda(x ; h, k)=-\left\langle(d \varphi)_{x} h,(d n)_{x} k\right\rangle, \quad x \in M, h, k \in T_{x}(M) .
$$

Thus $\Lambda$ determines a selfadjoint tensor field $\Gamma$ of type $(1,1)$ such that

$$
g(x ; \Gamma(x) h, k)=\Lambda(x ; h, k), \quad x \in M, h, k \in T_{x}(M),
$$

where $g$ denotes the Riemannian metric. Recall further that the mean curvature of $\varphi$ is defined by

$$
H=\frac{1}{2} \operatorname{tr} \Gamma \text {. }
$$

In this section we shall prove the following

$\mathrm{Th}$ e o r e m (Hopf). Let $M$ be a Riemannian 2-manifold which is diffeomorphic to $S^{2}$ and let $\varphi: M \rightarrow R^{3}$ be an isometric immersion with constant mean curvature. Then $\varphi$ is a diffeomorphism from $M$ onto a Euclidean 2-sphere in $\boldsymbol{R}^{3}$.

4.2. Gauss-Godazzi fields. A selfadjoint tensor field $\Theta$ of type $(1,1)$ on a Riemannian 2-manifold will be called a Gauss-Codazzi field, if

$$
\nabla_{X}(\Theta(Y))-\nabla_{Y}(\Theta(X))=\Theta([X, Y]), \quad X, Y \in \mathfrak{X}(M) .
$$

In particular, the tensor field $\Gamma$ determined by (4.1) is a Gauss-Codazzi field.

L e m m a I. A nonzero Gauss-Codazzi field, $\Theta$, with vanishing trace has only isolated zeros.

Proof. Set

$$
\hat{\Theta}=e^{\mu} \Theta
$$

where $\mu$ is a smooth function. Then $\hat{\Theta}$ satisfies the relation

$$
\nabla_{X}(\hat{\Theta} Y)-\nabla_{Y}(\hat{\Theta} X)=X(\mu) \hat{\Theta}(Y)-Y(\mu) \hat{\Theta}(X)+\hat{\Theta}([X, Y]) .
$$

Now choose a local Cauchy-Riemann frame $e_{1}, e_{2}$ and write

$$
\begin{aligned}
& \hat{\Theta} e_{1}=u e_{1}+v e_{2}, \\
& \hat{\Theta} e_{2}=v e_{1}-u e_{2} .
\end{aligned}
$$

Then (4.2) implies that

$$
e_{1}(v)-e_{2}(u)=e_{1}(\mu-f) v-e_{2}(\mu-f) u
$$

and 


$$
e_{1}(u)+e_{2}(v)=e_{1}(\mu-f) u+e_{2}(\mu-f) v,
$$

where $f=\ln \left|e_{1}\right|^{2}=\ln \left|e_{2}\right|^{2}$, as is easily checked by using 1.4 and 1.5. Now set

$$
\mu=f .
$$

Then these equations become

$$
\begin{aligned}
& e_{1}(v)=e_{2}(u), \\
& e_{2}(v)=-e_{1}(u) .
\end{aligned}
$$

It follows that $v+i u$ is a complex analytic function and so it can have only isolated zeros. Thus the same is true for $\hat{\Theta}$ and hence for $\Theta$.

$\mathrm{Cor}$ ollary. If $\Theta$ is a nonzero Gauss-Codazzi field with vanishing trace, then the function $\operatorname{det} \Theta$ has only isolated zeros.

$\mathrm{L}$ e $\mathrm{m} \mathrm{m}$ a II. Let $\Theta$ be a trace free Gauss-Codazi field and suppose that $\Theta(x) \neq 0$ for $x \in U$ (an open subset of $M$ ). Denote the positive eigenvalue of $\Theta(x)$ by $\lambda(x) \quad(x \in U)$. Let $Z$ be a smooth eigenvector field of $\Theta$ in $V \subset U$ such that $|Z|^{2}=\lambda^{-1}$. Then $Z$ is a $C R$-field.

Proof. Consider the 1-forms $\Phi_{Z}$ and $\Psi_{Z}$ (cf. section 1.2). We have to show that

$$
* \Phi_{Z}=\Psi_{Z} \text {. }
$$

Now the unit vector field

$$
X=\frac{1}{|Z|} Z=\sqrt{\lambda} Z
$$

satisfies

$$
\Theta(X)=\lambda X
$$

$X$ determines a 1 -form $\Psi_{X}$ (cf. section 1.2) such that

$$
\nabla_{Y} X=\Psi_{X}(Y) \cdot J(X), \quad Y \in \mathfrak{X}(M) .
$$

Since $\operatorname{tr} \Theta=0, J \circ \Theta+\Theta \circ J=0$. Thus $\Theta J X=-\lambda \cdot J X$. Putting $Y=J X$ in (4.2) we find that these relations imply that

$$
\left[J X(\lambda)-2 \lambda \cdot \Psi_{X}(X)\right] X+\left[X(\lambda)+2 \lambda \cdot \Psi_{X}(J X)\right] J X=0 .
$$

Thus (since $X, J X$ form a frame on $V$ )

$$
Y(\lambda)=-2 \lambda \cdot \Psi_{X}(J(Y)), \quad Y \in \mathfrak{X}(V) .
$$

Finally, observe that, by (1.5) and (1.6)

$$
\Phi_{Z}=-\frac{1}{2 \lambda} \delta \lambda \text { and } \Psi_{Z}=\Psi_{X}
$$

and thus (4.5) implies (4.4). 
4.3. The operator $\bar{\Theta}$. Let $\Theta$ be a nonzero trace free GaussCodazzi field and set $\dot{M}=M-\left\{a_{1}, \ldots, a_{r}\right\}$, where the $a_{i}$ are the zeros of $\Theta$ (cf. Lemma I). Then $\Theta$ induces a strong bundle map $\bar{\Theta}: P_{\dot{M}} \rightarrow P_{\dot{M}}$. Since $\Theta(x): T_{x}(\dot{M}) \rightarrow T_{x}(\dot{M})$ is a nonzero selfadjoint linear map with trace zero, there are precisely two (orthogonal) straight lines $\sigma_{1}(x)$ and $\sigma_{2}(x)$ in $T_{x}(\dot{M})$ spanned by its eigenvectors. These lines define crosssections $\sigma_{1}$ and $\sigma_{2}$ in $P_{\dot{M}}$ which satisfy

$$
\bar{\Theta}\left(\sigma_{i}\right)=\sigma_{i} \quad(i=1,2) .
$$

$\mathrm{L}$ e $\mathrm{m} \mathrm{m}$ a III. Let $\sigma$ be a cross-section in $P_{\dot{M}}$ such that $\bar{\Theta}(\sigma)=\sigma$ and let $a$ be one of the zeros of $\Theta$. Then

$$
j_{a}(\sigma)<0 \text {. }
$$

Proof. Assume first that $j_{a}(\sigma)$ is even. Then $\sigma$ lifts to a vector field $X$ in a neighbourhood $U$ of $a$ (cf. Proposition III, section 2.3). We may assume that $X$ is a unit vector field. Since

$$
\varrho(\Theta(X(x)))=\bar{\Theta}(\sigma(x))=\sigma(x)=\varrho(X(x)),
$$

it follows that $X$ is a unit eigenvector field of $\Theta$. Denote the corresponding eigenvalue at $x$ by $\lambda(x)$. Then, by Lemma II,

$$
Z=\frac{1}{\sqrt{\lambda}} X
$$

is a CR-field. Since $\lambda^{2}=-\operatorname{det} \Theta$, it follows that

$$
\lim _{x \rightarrow a}|Z|=\infty
$$

and so Proposition 1, section 2.1, implies that $j_{a}(Z)<0$. Thus, by formula $(2.4)$,

$$
j_{a}(\sigma)<0
$$

If $j_{a}(\sigma)$ is odd, choose a diffeomorphism $\varphi$ from $U$ onto the unit disk in the complex plane such that $\varphi(a)=0$ and set $\dot{U}=U-\{a\}$. Let $s: U \rightarrow U$ be the map which corresponds to the map $z \mapsto z^{2}$, $z \in C$, under $\varphi$. Then $(d s)_{x}: T_{x}(\dot{U}) \rightarrow T_{s(x)}(\dot{U})$ is a linear isomorphism for $x \in \dot{U}$. Thus $s$ induces a bundle map $\tilde{s}: P_{\dot{U}} \rightarrow P_{\dot{U}}$ and so $\sigma$ determines a cross-section $\sigma_{1}$ in $\dot{U}$ by (cf. section 2.2)

$$
\sigma_{1}(x)=\tilde{s}^{-1} \sigma(s(x)) \text {. }
$$


Finally, introduce a new Riemannian metric $g_{1}$ in $\dot{U}$ by $g_{1}=s^{*} g$ and consider the tensor field $\Theta_{1}$ given by

$$
\Theta_{1}(x)=(d s)_{x}^{-1} \Theta(s(x))(d s)_{x}, \quad x \in \dot{U} .
$$

Then $\Theta_{1}$ is a trace free Gauss-Codazzi field with respect to $g_{1}$ and

Since (cf. section 2.2)

$$
\bar{\Theta}_{1}\left(\sigma_{1}\right)=\sigma_{1}
$$

$$
j_{a}\left(\sigma_{1}\right)=\operatorname{deg}_{a} s \cdot j_{a}(\sigma)=2 j_{a}(\sigma)
$$

it follows from the first part of the proof that

Thus

$$
j_{a}\left(\sigma_{1}\right)<0 .
$$

$$
j_{a}(\sigma)<0
$$

4.4. Proof of the Hopf theorem. Let $\Gamma$ be the tensor field of type $(1,1)$ corresponding to the second fundamental form and set

$$
\Theta=\Gamma-H \cdot I
$$

( $I$ the unit tensor field). Then, since $H$ is constant, $\Theta$ is again a Gauss Codazzi field. Moreover.

$$
\operatorname{tr} \Theta=0
$$

We shall show that

$$
\Theta=0 \text {. }
$$

In fact, assume that $\Theta \neq 0$. Then, by Lemma 4.1, $\Theta$ has only finitely many zeros $a_{1}, \ldots, a_{r}(r \geqq 1)$. Set $\dot{M}=M-\left\{a_{1}, \ldots, a_{r}\right\}$. In view of section 4.3 there is a cross-section $\sigma$ in $P_{\dot{M}}$ such that

$$
\bar{\Theta}(\sigma)=\sigma .
$$

Hence, by Lemma III,

$$
j_{a_{i}}(\sigma)<0
$$

It follows that

$$
j_{\sigma}<0 .
$$

On the other hand, since $M$ is diffeomorphic to $S^{2}$, by the Gauss-Bonnet theorem,

$$
j_{\sigma}=2 \chi(M)=4 .
$$

Thus we have a contradiction and (4.7) follows. 
Now relation (4.6) implies via a standard result (cf. [6], p. 99) that $\varphi$ maps $M$ into a Euclidean sphere $S^{2}$ in $R^{3}$. Since $M$ is compact, this must be an onto map and hence a covering projection. Since $S^{2}$ is simply connected, it follows that $\varphi$ is a diffeomorphism.

\section{References}

[1] Greub, W., S. HAlperin, and R. VANSTONE: Connections, curvature, and cohomology. I. De Rham cohomology of manifolds and vector bundles. Pure and Applied Mathematics 47-I. Academic Press, New YorkLondon, 1972.

[2] -»- -»- -»- Connections, curvature, and cohomology. II. Lie groups, principal bundles, and characteristic classes. - Pure and Applied Mathematics 47-II. Academic Press, New York-London, 1973.

[3] HopF, H.: Über Flächen mit einer Relation zwischen den Hauptkrümmungen. Math. Nachr. 4, 1951, 232-249.

[4] Poincaré, H.: Sur les courbes définies par les équations différentielles (troisiéme partie). - J. Math. Pures Appl. (4) 1, 1885, 167-244. = Oeuvres de Henri Poincaré I. [Nouveau tirage.] Gauthier-Villars et Cie, Éditeurs, Paris, $1951,90-161$.

[5] DE Rham, G.: Variétés différentiables. Formes, courants, formes harmoniques. Actualités Sci. Ind. 1222. = Publ. Inst. Math. Univ. Nancago III, Hermann et Cie, Paris, 1955.

[6] STOKer, J. J.: Differential geometry. - Pure and Applied Mathematics 20. Wiley-Interscience, New York etc., 1969.

University of Toronto

Department of Mathematics

Toronto

Canada M5S 1Al

Received 7 November 1975 